\title{
Propagation of Scalar Fields in a Plane Symmetric Spacetime
}

\author{
Juliana Celestino $^{1}$ • Márcio E. S. Alves ${ }^{2}$ - F. A. Barone ${ }^{3}$
}

Received: 6 June 2016 / Published online: 3 October 2016

(c) Sociedade Brasileira de Física 2016

\begin{abstract}
The present article deals with solutions for a minimally coupled scalar field propagating in a static plane symmetric spacetime. The considered metric describes the curvature outside a massive infinity plate and exhibits an intrinsic naked singularity (a singular plane) that makes the accessible universe finite in extension. This solution can be interpreted as describing the spacetime of static domain walls. In this context, a first solution is given in terms of zero order Bessel functions of the first and second kind and presents a stationary pattern which is interpreted as a result of the reflection of the scalar waves at the singular plane. This is an evidence, at least for the massless scalar field, of an old interpretation given by Amundsen and Grøn regarding the behaviour of test particles near the singularity. A second solution is obtained in the limit of a weak gravitational field which is valid only far from the singularity. In this limit, it was possible to find out an analytic solution
\end{abstract}

Juliana Celestino

juliana.efei@gmail.com

Márcio E.S. Alves

marcio.alves@ict.unesp.br

F.A. Barone

fbarone@unifei.edu.br

1 Departmnt of Physics, Rio de Janeiro State University, R. São Francisco Xavier, 524 - Maracanã, Rio de Janeiro, RJ, 20550-900, Brazil

2 Instituto de Ciência e Tecnologia, UNESP - Univ Estadual Paulista, São José dos Campos, SP, 12247-004, Brazil

3 IFQ, University Federal of Itajubá, Av. BPS 1303, cep: 37500-903, Itajubá, Brazil for the scalar field in terms of the Kummer and Tricomi confluent hypergeometric functions.

Keywords Gravitational waves · Taub metric · Scalar field

\section{Introduction}

A variety of theories of fundamental physics predict the existence of scalar fields [1]. In cosmology, scalar fields are central to the implementation of the inflationary models [2, $3]$, and also in the development of alternatives to solve the puzzle of the present accelerated cosmic expansion $[4,5]$. All these subjects together with the recent discovery of the Higgs boson have been motivated a better understanding of the dynamical effects due to the coupling of scalar fields with gravity.

One of the most simple gravitational fields that we could conceive in Newtonian grounds is the homogeneous gravitational field produced by an infinite plate with superficial uniform mass density $\sigma$. The corresponding Newtonian potential is

$\varphi(z)=2 \pi G \sigma|z|$,

where we have supposed that the plate is located at the plane $z=0$.

The General Relativity version of this problem is the plane-symmetric solutions of Einstein's field equations. In 1951, Taub found the general vacuum solution [6]. A generalization to a spacetime with cosmological constant was found by Novotný and Horský [7], and the solution for a plane-symmetric scalar field was found by Singh [8]. Finally, a generalization of the Singh's solution with the presence of a massless plane symmetric scalar field and a cosmological constant was recently found by Vuille. [9] 
One particular solution which leads to the above potential in the Newtonian limit is the Rohrlich[10] solution that actually describes flat spacetime in a special coordinate system. On the other hand, in this article, we will consider the metric first found by Horský [11] which represents a truly curved spacetime. It is a particular solution of Einstein's equations of General Relativity with vanishing cosmological constant and vanishing surface charge density. For a detailed revision of general plane symmetric solutions of Einstein-Maxwell equations including discussions about the particular results, we refer the interested reader to the Ref. [12]. These solutions can be interpreted as describing the spacetime of domain walls.

Following a different approach, instead of searching for exact solutions of the Einstein's field equations, in this article, we consider the solutions of the equations of motion of "weak" scalar fields propagating in a plane symmetric background spacetime that describes the curvature outside an infinity massive plate located at $z=0$. We shall restrict to situations where the scalar field is said "weak" in the sense that the associated energy density and pressure of the field are weak enough to produce significant changes in the background curvature. As pointed out by Amundsen and Grøn, [12] this spacetime has the peculiarity of having an intrinsic naked singularity (a singular plane) in a finite distance from the massive plate that makes the accessible universe finite in extension. If this is true then a massless scalar field would be reflected or destroyed by the singular plane, i.e., it is not possible to escape from the gravity generated by the plate. In this context, the first solution we find is a particular solution of the equations of motion of a massless scalar field propagating perpendicular to the plate. The behaviour of the field in the vicinity of the massive plate and near the singular plane are then analysed. In a second approach, we aim to work out the solution of the equations of motion without restricting the direction of propagation of the scalar field. It is shown that this can be analytically achieved by focusing in the limiting case of weak gravitational field in the vicinity of the massive plate. In the last section, we present our conclusions and final remarks.

Along the paper, we use a method commonly employed in Quantum Field theory and in Optics. In this approach, non-linear equations are linearized for weak propagating fields.

\section{General Equations for the Propagation of Scalar Fields in a Planar Symmetric Spacetime}

The equation for a scalar field of mass $m$ coupled to gravity is

$\left(\square+m^{2}+\xi R\right) \phi=0$, where $\square=g^{\mu \nu} \nabla_{\mu} \nabla_{\nu}$ is the generalized D'Alembertian operator in curved spacetimes, $\nabla_{v}$ is the covariant derivative, $R$ is the curvature scalar, and $\xi$ is the coupling constant which specifies the "strength" of the interaction between $\phi$ and the gravitational field. There are two values of $\xi$ that are of particular interest, namely, 0 and $1 / 6$. If $\xi=0$, we say that the field $\phi$ is minimally coupled to gravity since the interaction occurs only through the modified D'Alembertian. If $\xi=1 / 6$, the scalar field is conformally coupled to gravity, i.e., the (2) is invariant under a conformal transformation of the metric.

It will be useful to consider the following identity

$g^{\mu \nu} \nabla_{\mu} \nabla_{\nu} \phi=\frac{1}{\sqrt{-\mathbf{g}}} \partial_{\mu}\left(\sqrt{-\mathbf{g}} g^{\mu \nu} \partial_{\nu} \phi\right)$,

where $\mathbf{g}=\operatorname{det}\left(g_{\mu \nu}\right)$ is the determinant of the metric $g_{\mu \nu}$.

In this article we consider the following metric [11]:

$$
\begin{aligned}
d s^{2}= & (1-3 g z)^{-2 / 3} d t^{2} \\
& -(1-3 g z)^{4 / 3}\left(d x^{2}+d y^{2}\right)-d z^{2},
\end{aligned}
$$

where $g$ is a constant. This metric describes a plane symmetric spacetime for $z \geq 0$ out of an infinity plate with uniform mass density $\sigma$ located at $z=0$.

The temporal component of the metric is related to the potential (1) in the Newtonian limit by

$g_{00} \simeq 1+\frac{2 \varphi}{c^{2}}$.

Therefore, the connection between the constant $g$ present in the metric (4) and $\sigma$ can be found by Taylor expanding the 00 component of the metric (4) to first order in $g z$

$g_{00} \simeq 1+2 g z$

and comparing (6) and (5) together with (1) is what leads to

$g=\frac{2 \pi G}{c^{2}} \sigma$,

where we identify $g c^{2}=2 \pi G \sigma$ as the Newtonian acceleration of gravity in the vicinity of the plate.

The fact that the spacetime described by the above metric is truly curved, can be seen by calculating the Kretschmann curvature invariant (see, e.g., Ref. [12]) which is not null. If it were null, the metric would not represent a curvature, but just the Minkowski spacetime described in a particular coordinate system. Furthermore, another important information can be obtained from this invariant; since the Kretschmann scalar diverges for $z_{+}=1 / 3 g$, we are led to conclude that there is a singular plane at $z_{+}$that, according to the physical interpretation given by Ref. [12], makes the universe finite in extension. In other words, a particle that resides above the massive plate is confined in the region $z \in\left[0, z_{+}\right]$. Notice also that in the plate at $z=0$, the metric (4) reduces to the Minkowski metric and, on the other hand, the curvature becomes more prominent as we approach $z_{+}$. 
At this point, it is important to have in mind the regime of validity of the calculations in this article. When we consider the metric (4) as representing the spacetime where the scalar field propagates, we are assuming that the resulting components of the energy-momentum tensor associated with $\phi$ are small enough to affect the spacetime curvature. In other words, we are assuming that "backreaction effects" of the field $\phi$ in the curvature are negligible in comparison with the effects produced by the massive plane in all over the space.

Now, let us substitute the metric (4) and its determinant $\mathbf{g}=-(1-3 g z)^{2}$ into the $(2)$ in order to write the following second order partial differential equation

$$
\begin{aligned}
(1-3 g z)^{5 / 3} \frac{\partial^{2} \phi}{\partial t^{2}} & -(1-3 g z)^{-1 / 3} \frac{\partial^{2} \phi}{\partial x^{2}} \\
& -(1-3 g z)^{-1 / 3} \frac{\partial^{2} \phi}{\partial y^{2}}-(1-3 g z) \frac{\partial^{2} \phi}{\partial z^{2}} \\
& +3 g \frac{\partial \phi}{\partial z}+m^{2}(1-3 g z) \phi=0
\end{aligned}
$$

Notice that in obtaining the above equation, we have used the fact that $R=0$ for the metric (4) since it was derived for an empty space, i.e., $T^{\mu \nu}=0$. Obviously, due to the presence of the plane, the energy-momentum tensor could not vanish at $z=0$ (for a detailed examination of the solution of Einstein's equations at the plane, we refer the reader to the Ref. [13]).

It is important to notice that (8) is linear for the field $\phi$, so any linear combination of independent solutions for $\phi$ is also a solution for this equation.

Supposing the field as a separable function

$\phi(t, \mathbf{x})=X(x) Y(y) Z(z) e^{-i \omega t}$,

we have

$$
\begin{aligned}
\frac{1}{X} \frac{d^{2} X}{d x^{2}} & +\frac{1}{Y} \frac{d^{2} Y}{d y^{2}} \\
& +(1-3 g z)^{4 / 3} \frac{1}{Z} \frac{d^{2} Z}{d z^{2}}-(1-3 g z)^{1 / 3} 3 g \frac{1}{Z} \frac{d Z}{d z} \\
& -m^{2}(1-3 g z)^{4 / 3}+(1-3 g z)^{2} \omega^{2}=0
\end{aligned}
$$

where $\omega$ is the angular frequency of the field measured at $z=0$.

For the two first terms, we have

$\frac{1}{X} \frac{d^{2} X}{d x^{2}}=-k_{x}^{2}, \quad \frac{1}{Y} \frac{d^{2} Y}{d y^{2}}=-k_{y}^{2}$,

and we are left with the following ordinary homogeneous differential equation for $Z(z)$,

$$
\begin{aligned}
\frac{d^{2} Z}{d z^{2}} & -\frac{3 g}{(1-3 g z)} \frac{d Z}{d z} \\
+ & {\left[(1-3 g z)^{2 / 3} \omega^{2}-\frac{k_{x y}^{2}}{(1-3 g z)^{4 / 3}}-m^{2}\right] Z=0 }
\end{aligned}
$$

where we interpret $k_{x y}=\sqrt{k_{x}^{2}+k_{y}^{2}}$ as the wave vector component which is parallel to the plate.

It is convenient to introduce a new variable $\bar{z}=3 g z$, where $\bar{z} \in[0,1]$, such that $\bar{z}=1$ locates the singular plane. For this variable, the (12) for $Z$ becomes

$$
\begin{aligned}
\frac{d^{2} Z}{d \bar{z}^{2}} & -\frac{1}{(1-\bar{z})} \frac{d Z}{d \bar{z}} \\
+ & {\left[\omega^{\prime 2}-\frac{k_{x y}^{\prime 2}}{(1-\bar{z})^{2}}-\frac{m^{\prime 2}}{(1-\bar{z})^{2 / 3}}\right](1-\bar{z})^{2 / 3} Z=0 }
\end{aligned}
$$

where $\omega^{\prime}=\frac{\omega}{3 g}, k_{x y}^{\prime}=\frac{k_{x y}}{3 g}$ and $m^{\prime}=\frac{m}{3 g}$.

We have not obtained a general analytical solution for the above equation but in the following sections we present solutions for two particular situations. The first one is the case of a massless scalar field propagating in the $+z$ direction, while the second one is a solution for small $\bar{z}$, i.e., when the field is propagating in a region near the massive plate and far away from the singular plane.

\section{Analytic Solution for Zero Mass and Perpendicular Propagation}

Considering the (13) for the particular case of zero mass $m=0$ and choosing the field propagation in the perpendicular direction to the plate, i.e., $k_{x y}=0$ with the functions $X$ and $Y$ constants, we have

$\frac{d^{2} Z}{d \bar{z}^{2}}-\frac{1}{(1-\bar{z})} \frac{d Z}{d \bar{z}}+\omega^{\prime 2}(1-\bar{z})^{2 / 3} Z=0$,

which has the following general solution in terms of the zero order Bessel and Neumann functions

$Z(u)=A J_{0}(u)+B N_{0}(u)$,

with $u=\frac{3}{4} \omega^{\prime}(\bar{z}-1)^{4 / 3}$. The function $J_{0}(u)$ has always a finite value, for every $\omega$, in the region between the massive plate and the singular plane. However, the Neumann function $N_{0}(u)$ decreases abruptly near the singular plane and goes to $-\infty$ as $\bar{z} \rightarrow 1$. Notice that the singularity appearing in the function $N_{0}$ does not mean that we should discard this solution, but this is an expected behaviour of the scalar field at $\bar{z}=1$ since the spacetime is singular at that plane as it is evidenced by the evaluation of the Kretschmann scalar as mentioned previously. Therefore, the propagation of the scalar field turns out to be a useful tool to identify some properties of the spacetime like the presence of singularities.

A natural question the reader can raise relies on the fact that a singular solution for $\phi$ could induce non-negligible modifications in the metric and, so, produce backreaction effects. This problem can be solved in two alternative ways. The first one is simply to discard the divergent solutions 
in (15), by setting $B=0$. The second approach is more subtle and consists in adjusting the initial conditions of the scalar field in such a way that its contribution to the metric is negligible in comparison with the ones produced by the massive plane. Let us start by the second approach.

The constants $A$ and $B$ can be found by imposing initial conditions for the function $\phi$ and its first derivative at the massive plate, located at $z=0$. Hence, let us consider for instance,

$\phi(z=0, t=0)=\phi_{0},\left.\quad \frac{\partial \phi}{\partial z}\right|_{z=0, t=0}=0$.

By using conditions (16) as well as the Wronskian for Bessel functions of (15), we obtain

$A_{\omega}=-\phi_{0} \frac{3 \pi \omega^{\prime}}{8} N_{1}\left(3 \omega^{\prime} / 4\right)$

and

$B_{\omega}=\phi_{0} \frac{3 \pi \omega^{\prime}}{8} J_{1}\left(3 \omega^{\prime} / 4\right)$.

The final real solution for the scalar field is

$$
\begin{aligned}
\phi(t, z)= & \phi_{0} \frac{3 \pi \omega^{\prime}}{8} \cos \omega t\left[J_{1}\left(3 \omega^{\prime} / 4\right) N_{0}(u)\right. \\
& \left.-N_{1}\left(3 \omega^{\prime} / 4\right) J_{0}(u)\right] .
\end{aligned}
$$

The constant $\phi_{0}$ must be small enough in such a way that any possible metric effects induced by the scalar field are negligible in comparison with the ones produced by the massive plane. In this way, we shall not have backreaction effects.

A similar result can be found for the case $\omega=0$ and $k_{x y} \neq 0$, for which the solution is given in terms of modified Bessel functions, as follows,

$\phi(t, z)=\cos \omega t\left[C_{1} I_{0}(v)+C_{2} K_{0}(v)\right]$

where we defined $v=3 k_{x y}^{\prime}(\bar{z}-1)^{1 / 3}$ and $C_{1}$ and $C_{2}$ are constants. Also, for this case, there is a singularity in the plane $\bar{z}=1$.

In the Fig. 1, we show the spatial behaviour of the scalar field $\phi$ of the solution (19) at $t=0$ for three values of frequency. Note that the solution (19) represents a stationary pattern for the scalar field. A stationary wave solution can be particularly noticed for high frequency waves $\omega^{\prime} \gg 1$ in the vicinity of the massive plate $\bar{z} \ll 1$ (it is worth to notice that $\omega^{\prime} \gg 1$ implies $\omega \gg c / z_{+}$, where $z_{+}=1 / 3 g$; that means that the period of the wave as measured by a clock located at $z=0$ is much shorter than the time it takes light to travel from the massive plate to the singular plane $T_{+}=$ $z_{+} / c$, also $\bar{z} \ll 1$ implies that the distance to the planar singularity is much greater than the considered distance to the plate $z \ll z_{+}$), and then by using the asymptotic forms of the Bessel functions of the first and second kind for large real arguments [14]

$J_{0}(u) \sim \sqrt{\frac{2}{\pi u}} \cos \left(u-\frac{\pi}{4}\right), \quad N_{0}(u) \sim \sqrt{\frac{2}{\pi u}} \sin \left(u-\frac{\pi}{4}\right)$,

we find

$\phi(t, z) \simeq \phi_{0}(1+4 g z) \cos \omega z \cos \omega t$.

The position of the nodes for this asymptotic limit depends on $\omega$ and are given by

$z_{n}=\frac{\pi(1+2 n)}{2 \omega}, \quad n=0,1,2, \ldots$

By taking $\bar{z} \rightarrow 0$, we note that the above expression is a solution of the usual wave equation in the flat space; this is in accordance with the fact that the metric we are using reduces to the Minkowski metric at the massive plane. Also, for the same limit, it is possible to define the $z$ component of the wave vector by the following eigenvalue equation

$\lim _{z \rightarrow 0}\left(\frac{\partial^{2} \phi(t, z)}{\partial z^{2}}\right)=-k_{z}^{2} \phi(t, z)$,

from which we identify $k_{z}=\omega$ and, by restoring physical units, we find the phase velocity at the plate $\omega / k_{z}=c$.

How do we physically interpret the obtained solution? Our stationary solution suggests that we have a combination of two waves, one travelling to the right and the other travelling to the left after being reflected by the singular plane at $z_{+}$. For the asymptotic solution (22), we can write explicitly

$\phi(t, z)=\phi_{-}(z-t)+\phi_{+}(z+t)$,

where

$\phi_{-}(z-t)=(1+4 g z) \frac{\phi_{0}}{2} \sin \left[\omega(z-t)+\frac{\pi}{2}\right]$,

and

$\phi_{+}(z+t)=(1+4 g z) \frac{\phi_{0}}{2} \sin \left[\omega(z+t)+\frac{\pi}{2}\right]$.

This is a proof of the interpretation given in Ref. [12] by Amundsen and Grøn, which comes from the analysis of the geodesic equation of particles in the spacetime described by the metric (4). According to those authors, only massless particles, like photons, can reach the singular plane at $z_{+}$ and then they fall back. With the geodesic equation it is possible to show that photons reach $z_{+}$but it is not possible to show that they are reflected, this was a matter of interpretation at that time. The same happens for massive particles, but in this case there is a maximum height $z_{M}$ for which $z_{M}<z_{+}$. The solution we have obtained gives a proof, at least for a real weak scalar field, that massless particles are reflected by the singularity. We could think that the same would happen for other massless fields, like the abelian vector field which represents the propagation of photons. 
Fig. 1 Behaviour of the scalar field $\phi$ at the time $t=0$ between the massive plate at $\bar{z}=0$ and the singular plane at $\bar{z}=1$ for three values of frequency

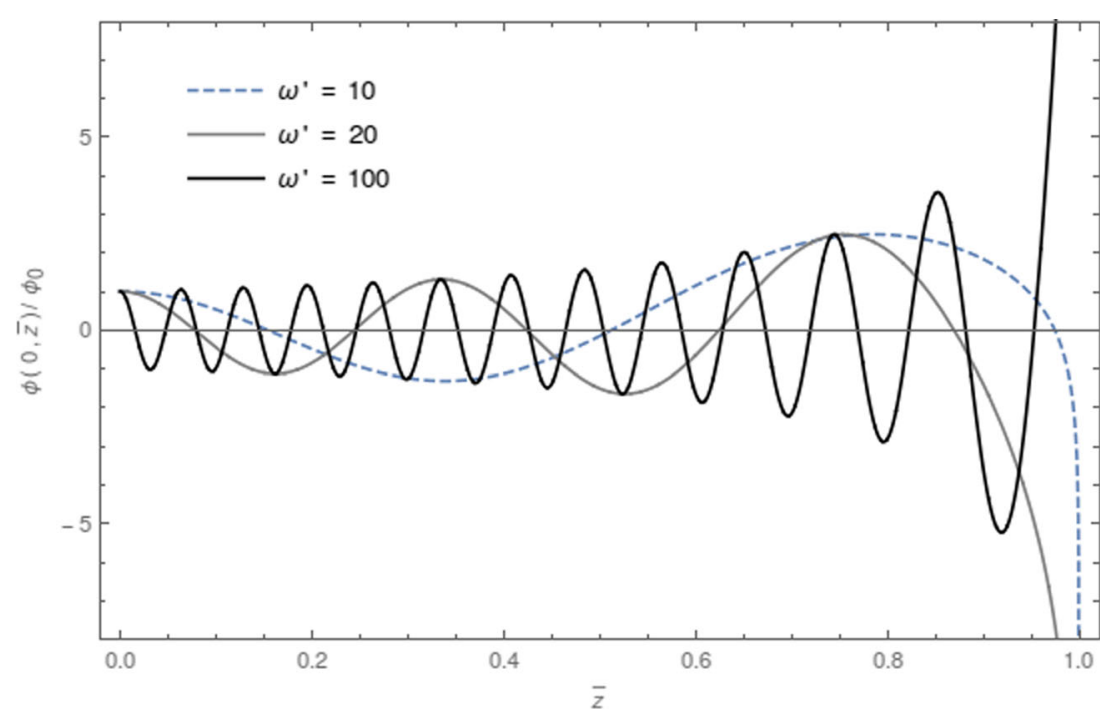

Also, in analysing the spacetime structure our solution reinforces the interpretation that the physical universe outside the massive plate has a finite extension in the $z$ coordinate.

A quantity of particular interest is the scalar field energy distribution in the region between $\bar{z}=0$ and 1 . This quantity can be evaluated from the 00 component of the energymomentum tensor of the scalar field which, in its general form for curved spacetimes, is [15]

$$
\begin{aligned}
T_{\mu \nu}= & (1-2 \xi) \nabla_{\mu} \phi \nabla_{\nu} \phi+(2 \xi-1 / 2) g_{\mu \nu} \nabla_{\lambda} \phi \nabla^{\lambda} \phi \\
& -2 \xi \phi \nabla_{\mu} \nabla_{\nu} \phi+2 \xi \phi \square \phi g_{\mu \nu}+\xi G_{\mu \nu} \phi^{2}-\frac{1}{2} g_{\mu \nu} m^{2} \phi^{2}
\end{aligned}
$$

This expression is greatly simplified if we remember that for the case we are studying $G_{\mu \nu}=0, m=0$ and therefore $\square \phi=0$. Also, for simplicity, we will perform the calculations only for the minimally coupled case $\xi=0$ (note that although the coupling constant $\xi$ does not appear in the field equations for $\phi$ since $R=0$, in general it affects the components of the energy-momentum tensor). With all these considerations, the energy-momentum tensor reduces to

$T_{\mu \nu}=\nabla_{\mu} \phi \nabla_{\nu} \phi-\frac{1}{2} g_{\mu \nu} \nabla_{\lambda} \phi \nabla^{\lambda} \phi$.

Let us define the averaged energy density

$\rho=\left\langle T_{00}\right\rangle$,

where the brackets denote time average over one period. After performing the calculations we obtain

$$
\begin{aligned}
\rho_{\omega^{\prime}}(z)= & \frac{\omega^{\prime 2}}{4}\left\{\left[A_{\omega^{\prime}} J_{0}(u)+B_{\omega^{\prime}} N_{0}(u)\right]^{2}\right. \\
& \left.+\left[A_{\omega^{\prime}} J_{1}(u)+B_{\omega^{\prime}} N_{1}(u)\right]^{2}\right\} .
\end{aligned}
$$

It is important o enphasize that the constants $A_{\omega}$ and $B_{\omega}$ must be small enough in such a way that the contribution to the metric field induced by the scalar field are negligible in comparison with the contribution produced by the massive plane.

In Fig. 2 we can see the spatial distribution of the scalar field energy density between the massive plate and the naked planar singularity. Near $\bar{z}=0$, the function $\rho(\bar{z})$ has a smooth increase, but it increases very fast when it approaches the singularity and goes to infinity for $\bar{z}=1$. Remember that we have assumed that the energy density of the scalar field was small enough to affect the spacetime geometry. As we can see, although that could be true near the massive plate, the energy density gained by the scalar field from the prominent curvature near $\bar{z}=1$ makes $\rho(\bar{z})$ arbitrarily large in such a way that $T_{\mu \nu}$ could not be disregarded in order to determine the spacetime metric. A new metric that results from the solution of the Einstein's field equations with the $T_{\mu \nu}$ given by (29) as a source term was first obtained by Singh [8] and a generalization with cosmological constant was recently obtained by Vuille [9]. It is interesting to notice that the Singh solution contains the same singularity that appears in the metric we are considering; therefore, there are no qualitative changes in the spacetime structure when a non-null energy-momentum tensor for the scalar field is assumed.

As mentioned previously, one could discard the divergent solution by setting $B=0$ in (15). In this case, the solution for (14) reads

$Z(u)=A J_{0}(u)$.

The constant $A$ is determined by the initial conditions. As an example, let us use the ones of (16). In this case, we have that $A=\phi_{0}$ and the possible values for the frequency $\omega$ become discrete, given by

$\omega^{\prime}=\frac{4}{3} \alpha_{1, n}$, 
Fig. 2 Distribution of the energy density $\rho(\bar{z})$ in the region between the massive plate and the singular plane

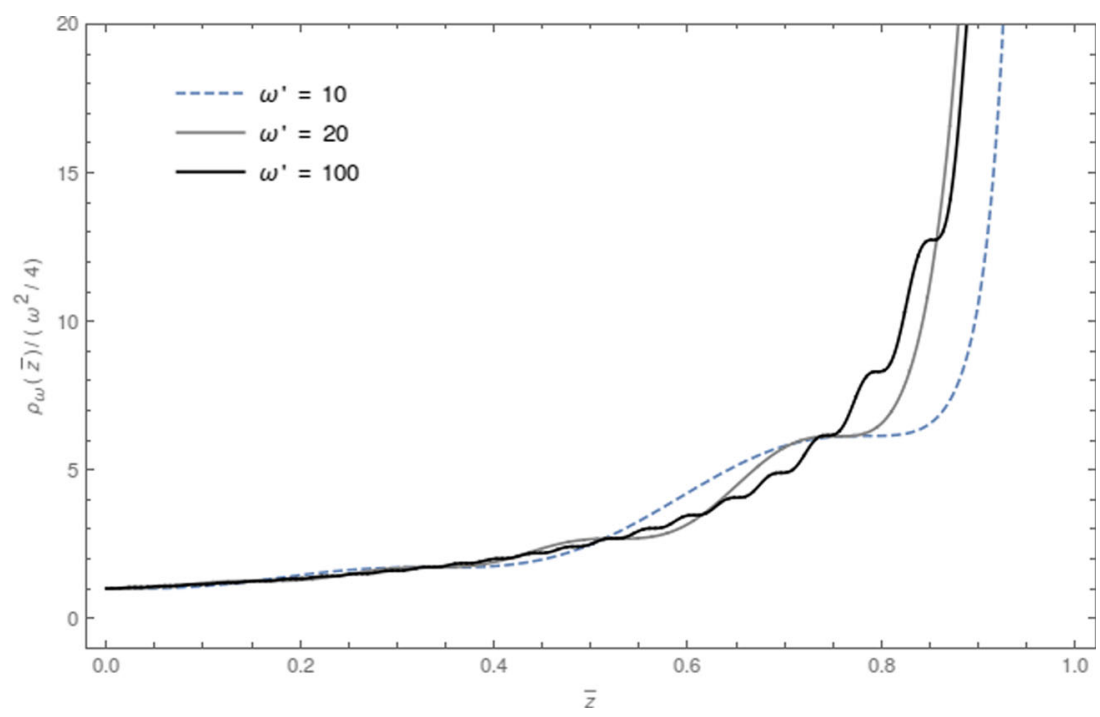

where $\alpha_{1, n}$ stands for the roots of the Bessel function $J_{1}$, namely, $J_{1}\left(\alpha_{1, n}\right)=0$.

The corresponding averaged energy density (30) is

$\rho_{\omega^{\prime}}(z)=\frac{\omega^{\prime 2}}{4} A_{\omega^{\prime}}^{2}\left[\left(J_{0}(u)\right)^{2}+\left(J_{1}(u)\right)^{2}\right]$.

In Fig. 3, we have a plot for $\rho$ given in (34) with $\alpha_{1,2} \cong$ $7.01559, \alpha_{1,5} \cong 16.4706$ and $\alpha_{1,10} \cong 32.1897$. We can see that on the singular plane, $\bar{z}=1$, the energy density (3) is always non-divergent. For higher zeros $\alpha_{1, n}$, we have more oscillations for the energy density, which exhibits its higher values in the vicinity of the singular plane.

\section{Solution in the Vicinity of the Massive Plate}

In this section, we consider the scalar field propagation in the vicinity of the massive plane and far away from the singular plane. In this situation, we must have $\bar{z}<<1$, so we can linearize (13) in $\bar{z}$ as follows,

$$
\begin{gathered}
\frac{d^{2} Z}{d \bar{z}^{2}}-(1+\bar{z}) \frac{d Z}{d \bar{z}}+\left[\omega^{\prime 2}-k_{x y}^{\prime 2}-m^{\prime 2}\right. \\
\left.-\left(\frac{2}{3} \omega^{\prime 2}+\frac{4}{3} k_{x y}^{\prime 2}\right) \bar{z}\right] Z=0 .
\end{gathered}
$$

It is important to mention that (35) is valid just in the vicinity of the massive plane (far away from the singular plane), and so, their solutions must be considered only in this region.

The solution for (35) is given in terms of the so-called Kummer functions of first and second types, $M(a, b ; j)$ and $U(a, b ; j)$, respectively, as follows,

$$
\begin{aligned}
Z= & e^{\frac{-2}{3} \bar{z} \omega^{2}-\frac{4}{3} \bar{z} k_{x y}^{\prime 2}}\left[C_{1} M(a, 1 / 2 ; R(\bar{z}))\right. \\
& \left.+C_{2} U(a, 1 / 2 ; R(\bar{z}))\right],
\end{aligned}
$$

where $C_{1}$ and $C_{2}$ are constants determined by the initial conditions and we defined the constant

$a=\frac{m^{\prime 2}}{2}-\frac{1}{18}\left[16 k_{x y}^{\prime 4}+\left(3+16 \omega^{\prime 2}\right) k_{x y}^{\prime 2}+4 \omega^{\prime 4}+15 \omega^{\prime 2}\right]$,

and the function

$R(\bar{z})=\frac{\left(3 \bar{z}+4 \omega^{\prime 2}+8 k_{x y}^{\prime 2}+3\right)^{2}}{18}$.

Equation (35) is valid only for small values of $\bar{z}$, so we must consider the solution (36) for $\bar{z}<<1$. For this task, we use the fact that in the first order in $\bar{z}$, we have

$$
\begin{aligned}
M(a, 1 / 2 ; R(\bar{z})) \cong & M\left(a, 1 / 2, \alpha^{2} / 18\right) \\
& +\frac{2}{3} a \alpha M\left(a+1,3 / 2, \alpha^{2} / 18\right) \bar{z},
\end{aligned}
$$

$$
\begin{aligned}
U(a, 1 / 2 ; R(\bar{z})) \cong & U\left(a, 1 / 2, \alpha^{2} / 18\right) \\
& +\bar{z} \sqrt{\pi}\left[\frac{2}{3} \frac{a \alpha M\left(a+1,3 / 2, \alpha^{2} / 18\right)}{\Gamma(a+1 / 2)}\right. \\
& -\frac{\sqrt{2}}{27} \frac{\alpha^{2}(1+2 a)}{\Gamma(a)} M\left(a+\frac{3}{2}, \frac{5}{2}, \frac{\alpha^{2}}{18}\right) \\
& \left.\left.-\frac{\sqrt{2}}{\Gamma(a)} M\left(a+1 / 2,3 / 2, \alpha^{2} / 18\right)\right],(40)\right]
\end{aligned}
$$

where $\Gamma$ stands for the Euler Gamma function, and we defined the quantity

$\alpha=4 \omega^{\prime 2}+8 k_{x y}^{\prime 2}+3$. 


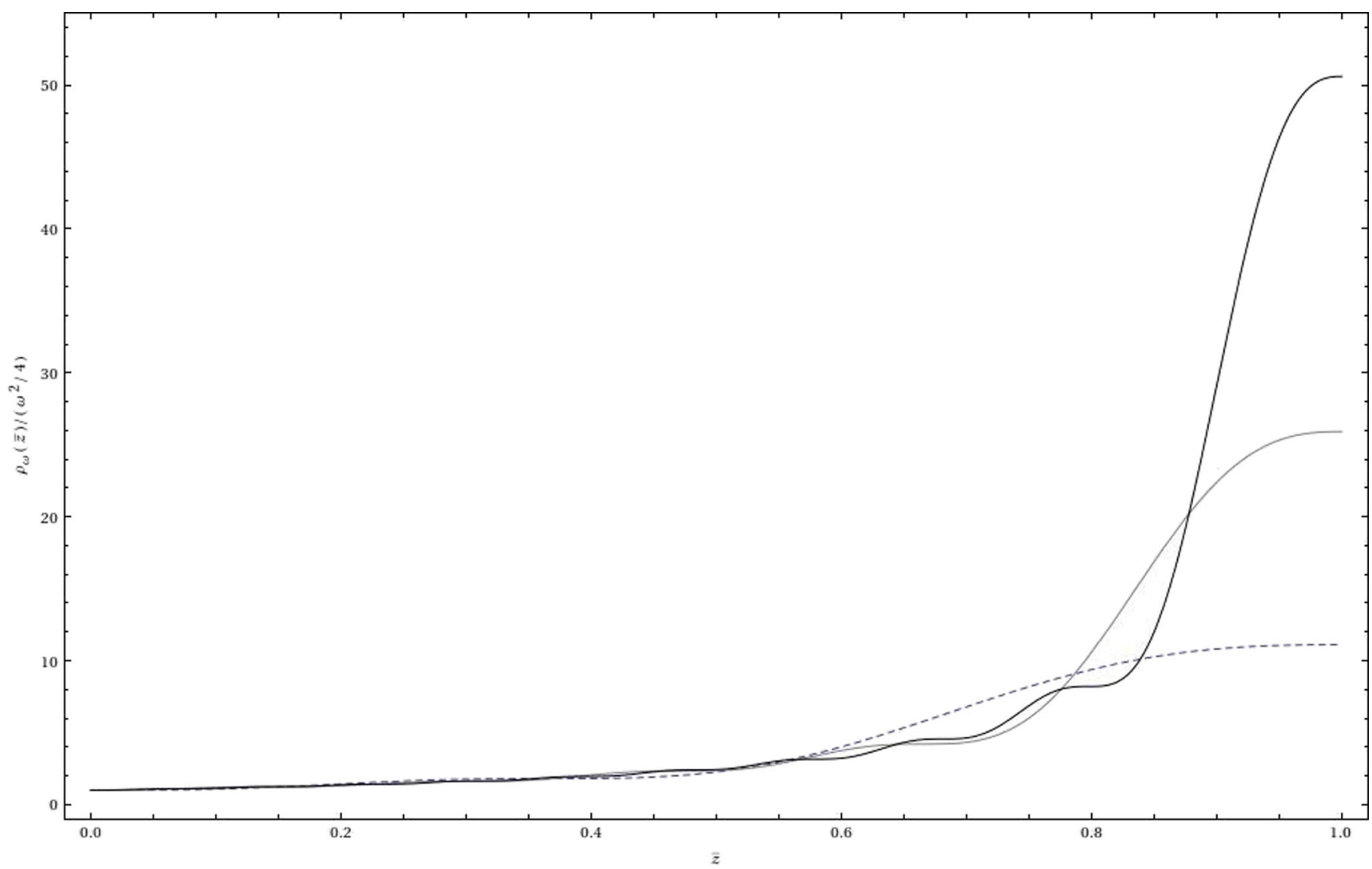

Fig. 3 Distribution of the energy density $\rho(\bar{z})$ in the region between the massive plate and the singular plane for non-singular solutions. We have used $\alpha_{1,2} \cong$. 01559 (dashed line), $\alpha_{1,5} \cong 16.4706$ (grey line) and $\alpha_{1,10} \cong 32.1897$.black line)

As we said, the constants $C_{1}$ and $C_{2}$ are determined by the initial conditions of the propagating field $\phi(x)$. Since we are interested only in the behaviour of the field, we shall not impose specific boundary conditions for the scalar fields

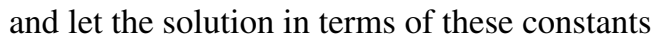

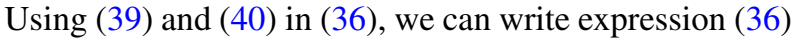

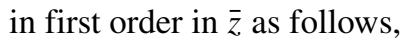

$$
\begin{aligned}
Z(z) \cong & e^{\frac{-2}{3} \bar{z} \omega^{2}-\frac{4}{3} \bar{z} k_{x y}^{\prime 2}}\left[\Phi_{0}\left(\omega, k_{x y}\right)+\Phi_{1}\left(\omega, k_{x y}\right) \bar{z}\right] \\
\cong & {\left[1-\left(2 \omega^{\prime 2} / 3+4 k_{x y}^{\prime 2} / 3\right) \bar{z}\right]\left[\Phi_{0}\left(\omega, k_{x y}\right)\right.} \\
& \left.+\Phi_{1}\left(\omega, k_{x y}\right) \bar{z}\right] \\
\cong & \Phi_{0}\left(\omega, k_{x y}\right)+\left[\Phi_{1}\left(\omega, k_{x y}\right)\right. \\
& \left.-\left(2 \omega^{\prime 2} / 3+4 k_{x y}^{\prime 2} / 3\right) \Phi_{0}\left(\omega, k_{x y}\right)\right] \bar{z}
\end{aligned}
$$

where we expanded the exponential and defined the func-

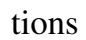

$\Phi_{0}\left(\omega, k_{x y}\right)=C_{1} M\left(a, 1 / 2, \alpha^{2} / 18\right)+C_{2} U\left(a, 1 / 2, \alpha^{2} / 18\right)$

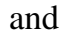

$$
\begin{aligned}
\Phi_{1}\left(\omega, k_{x y}\right)= & C_{1} \frac{2}{3} a \alpha M\left(a+1,3 / 2, \alpha^{2} / 18\right) \\
& +C_{2} \sqrt{\pi}\left(\frac{2}{3} \frac{a \alpha M\left(a+1,3 / 2, \alpha^{2} / 18\right)}{\Gamma(a+1 / 2)}\right. \\
& -\frac{\sqrt{2}}{27} \frac{\alpha^{2}(1+2 a)}{\Gamma(a)} M\left(a+3 / 2,5 / 2, \alpha^{2} / 18\right) \\
& \left.-\sqrt{2} \frac{1}{\Gamma(a)} M\left(a+1 / 2,3 / 2, \alpha^{2} / 18\right)\right),
\end{aligned}
$$

which do not depend on $\bar{z}$.

With the (43), one can write the propagating scalar field

$$
\begin{aligned}
\phi= & \operatorname{Re}\left[e ^ { - i ( \omega t + k _ { x } x + k _ { y } y ) } \left[\Phi_{0}\left(\omega, k_{x y}\right)\right.\right. \\
& \left.\left.+\left(\Phi_{1}\left(\omega, k_{x y}\right)-\left(2 \omega^{\prime 2} / 3+4 k_{x y}^{\prime 2} / 3\right) \Phi_{0}\left(\omega, k_{x y}\right)\right) \bar{z}\right]\right]
\end{aligned}
$$

Substituting the solution (45) in the 00 component of the tensor (29) we have

$$
T_{00}(t, x, y, \bar{z})=[F(t, x, y)+G(t, x, y) \bar{z}],
$$


where we defined the functions

$$
\begin{aligned}
F(t, x, y)= & \frac{1}{2}\left\{\left(\omega^{2}+k_{x}^{2}+k_{y}^{2}\right) \Phi_{0}^{2}\left(\omega, k_{x y}\right)\right. \\
& \times \sin ^{2}\left(\omega t-k_{x} x-k_{y} y\right)+(3 g)^{2}\left[\Phi_{1}\left(\omega, k_{x y}\right)\right. \\
& \left.\left.-\beta \Phi_{0}\left(\omega, k_{x y}\right)\right] \cos ^{2}\left(\omega t-k_{x} x-k_{y} y\right)\right\},(47)
\end{aligned}
$$

$$
\begin{aligned}
G(t, x, y)= & \frac{(3 g)^{2}}{3}\left[\Phi_{1}\left(\omega, k_{x y}\right)-\beta \Phi_{0}\left(\omega, k_{x y}\right)\right] \\
& \times \cos ^{2}\left(\omega t-k_{x} x-k_{y} y\right) \\
& +\left\{\left(k_{x}^{2}+k_{y}^{2}\right) \Phi_{0}^{2}\left(\omega, k_{x y}\right)\right. \\
& +2\left(\omega^{2}+k_{x}^{2}+k_{y}^{2}\right) \Phi_{0}\left(\omega, k_{x y}\right) \\
& \left.\times\left[\Phi_{1}\left(\omega, k_{x y}\right)-\beta \Phi_{0}\left(\omega, k_{x y}\right)\right]\right\} \\
& \times \sin ^{2}\left(\omega t-k_{x} x-k_{y} y\right)
\end{aligned}
$$

and the constant

$\beta=2 \omega^{\prime 2} / 3+4 k_{x y}^{\prime 2} / 3$.

From (46), (47), (48) and (49), we can see that the energy density of the scalar field (45) exhibits a plane wave-like behaviour on the parallel coordinates to the massive plane ( $x, y$ coordinates). Similar calculations show that the other components of $T^{\mu \nu}$ exhibit an analogous behaviour to that one obtained for $T^{00}$, with an increase along the $z$ direction, as expected from the previous section.

\section{Conclusion}

In this article we worked out the solutions for the equation of motion of a scalar field minimally coupled to gravity and propagating in a plane symmetric spacetime.

We restricted ourselves to weak field configurations, where backreaction effects are negligible.

Our first solution was obtained considering the scalar field propagating along the direction perpendicular to the plane. Written in terms of the zero order Bessel functions of the first and second kind, this solution showed that the scalar field presents a stationary pattern which, in the vicinity of $z=0$ and by considering the asymptotic limit of high frequencies, can be expressed in a simple manner in terms of stationary sine waves with linearly increasing amplitude. We interpreted this solution by saying that the scalar field waves are reflected by the singular plane at $z=z_{+}$. In this point of view, the result can be understood as a proof of the argumentation given by Amundsen and Grøn [12] in order to interpret what happens when massless particles approaches the singularity. Since massless particles can not escape from the gravitational field produced by the massive plate, we can say that the accessible Universe is spatially finite. An interesting extension of this solution would be to find either an analytic or a numerical solution for the massive scalar field propagation. For this case, it is expected that the scalar field would not reach the singular plane $z_{+}$, but it would be reflected in a maximum position $z_{M}<z_{+}$. Some care must be taken with this solutions because they are singular at the plane $\bar{z}=1$. The contribution of the energy momentum of the scalar field on the metric must be much lower that the influence of the massive plane on the metric. This condition is attained by imposing appropriated boundary conditions on the scalar field in such a way to keep the weak field aproximation always valid, where the effects of back reaction are negligible.

Our solution becomes singular at $z_{+}$where the energy density of the scalar field is infinity. Furthermore, near the singularity, the solution is no longer valid since it is not possible to keep the initial assumption that the presence of the scalar field does not change the spacetime structure. In this situation, a new metric that results from the solution of the Einstein's field equations with the $T_{\mu \nu}$ given by (29) as a source term should be found. Such an exact solution was first obtained by Singh[8] and a generalization with cosmological constant was recently obtained by Vuille. [9] It is interesting to notice that the Singh solution contains the same singularity that appears in the metric we are considering, therefore there are no qualitative changes in the spacetime structure when a non-null energy-momentum tensor for the scalar field is assumed.

As a special case, we restricted our results only to nondivergent solutions, where the allowed frequencies became discret.

We are left also with the question of what happens with the energy density, and with the other components of the energy-momentum tensor, for different coupling parameters $\xi$. Remember that in spite of the fact that $\xi$ does not contribute to the scalar field solution of the equation of motion (because the considered metric gives $R=0$ ), it contributes to the definition of the energy-momentum tensor in curved spacetimes given by (28).

Finally, we also found a solution for the scalar field considering the plane symmetric metric in the limit of a weak gravitational field. In this limit, it was possible to find out an analytic solution for the scalar field without restricting the direction of propagation. In this case, our results are valid only in the vicinity of the massive plane and were obtained in terms of the Kummer and Tricomi confluent hypergeometric functions. As expected from the previous result, the amplitude of the scalar field and the corresponding energy density increase linearly in the $z$ direction.

More general solutions could be found by writing down the full action of the problem and solving the coupled dynamical equations. In this way, back reaction effects due to the divergences of the solutions could be taken into account. 
Acknowledgments JC would like to thank to FAPEMIG for financial support. MESA would like to thank the Brazilian agency FAPESP for financial support (grant 13/26258-4).F.A. Barone would like to thank to CNPq financial support (grant: 311514/2015-4, 484736/2012-4).

\section{References}

1. M.B. Green, J.H. Schwarz, E. Witten, Superstring Theory (Cambridge University Press, Cambridge, England, 1987)

2. A.H. Guth, Inflationary universe: A possible solution to the horizon and flatness problems. Phys. Rev. D 23, 347 (1981)

3. K.A. Olive, Inflation. Phys. Rep. 190, 307 (1990)

4. S. Perlmutter et al., Measurements of Omega and Lambda from 42 high-redshift supernovae. Astron. J. 517, 565 (1999)

5. A.G. Riess et al., Observational evidence from supernovae for an accelerating universe and a cosmological constant. Astron. J. 116, 1009-1038 (1998)

6. A.H. Taub, Empty space-times admitting a three parameter group of motions. Ann. Math. 53, 472 (1951)
7. J. Novotný, J. Horský, On the plane gravitational condensor with the positive gravitational constant,. Czech. J. Phys. B 24, 718-723 (1974)

8. T. Singh, A plane symmetric solution of Einstein's field equations of general relativity containing zero-rest-mass scalar fields. Gen. Relativ. Gravit. 5, 657-662 (1974)

9. C. Vuille, Exact solutions for the massless plane symmetric scalar field in general relativity, with cosmological constant. Gen. Relativ. Gravit. 39, 621-632 (2007)

10. F. Rohrlich, The Principle of equivalence. Ann. Phys. 22, 169-191 (1963)

11. J. Horský, The gravitational field of planes in general relativity. Czech. J. Phys. B 18, 569-583 (1968)

12. P.A. Amundsen, $\varnothing$. Grn, General static plane-symmetric solutions of the Einstein-Maxwell equations,. Phys. Rev. D 27, 1731-1739 (1983)

13. P. Jones et al., The general relativistic infinite plane,. Am. J. Phys. 76, 73-78 (2008)

14. M. Abramowitz, A. Irene, eds, Handbook of Mathematical Functions (Dover, New York, 1965), p. 364

15. N.D. Birrell, P.C.W. Davies, Quantum Fields in Curved Space (Cambridge University Press, Cambridge, England, 1982), p. 87 